\title{
Greedy Algorithm for Image Compression in Image Processing
}

\author{
Priya Bajpai \\ Department of Computer \\ Science \& Engineering \\ PG Scholar,SRMCEM \\ Lucknow
}

\author{
Pankaj Kumar, PhD \\ Department of Computer \\ Science \& Engineering \\ Assistant Professor, SRMCEM \\ Lucknow
}

\author{
Raj Gaurang Tewari, PhD \\ Department of Computer \\ Science \& Engineering \\ Assistant Professor, SRMCEM \\ Lucknow
}

\begin{abstract}
The image compression is the technique if image processing which is applied to reduce size of the input image. The image compression is broadly classified into lossy and lossless type of compression. The WDR algorithm is the lossy type of compression. In this paper, improvement in the WDR algorithm is been proposed and improvement is based on decision tree algorithm. The proposed algorithm is been implemented in MATLAB and it is been analyzed that compression ratio and PSNR is increased in the proposed algorithm.
\end{abstract}

\section{Keywords}

WDR, PSNR, Compression ratio, lossy, lossless

\section{INTRODUCTION}

The enhancement of raw images gathered from cameras, satellites, air ships or from daily routines for various application sis known as image processing. The enhancement of image through electrical measures is done within the analog type of image processing methods. Television images are the most common example of these types of images [1].In this case, digital computers are utilized to process the image. The image will be converted to digital form utilizing a scanner - digitizer and after that

Process it. It is defined as the subjecting numerical representations of objects to a series of operations keeping in mind the end goal to acquire a fancied result. The system incorporates Image processing module and knowledge management and prediction module. Image processing module deals with operations on image up to pattern acknowledgment [2].

The knowledge about medical palmistry is fed into the system in second module. In this module, sample patterns are fed into knowledge base. Finally these two modules consolidate their work items, and system creates last output, that is prediction. Classification between the objects is simple task for humans yet it has turned out to be a complex problem for machines. The raise of high-capacity computers, the availability of high quality and low-priced video cameras, and the increasing requirement for automatic video analysis has generated an interest in object classification algorithms [3]. Within a simple classification scenario, the camera is placed at the higher zone of the area to be monitored. The collected images are then to be processed [4]. Image compression is a technique that is proposed for storing or retrieving data from the image in an efficient manner by ensuring that the irrelevance and redundancy of the data present within the image is not reduced. The size of the image is reduced in bytes within the image compression method by making sure that the quality of the image is not compromised. This helps in storing more number of images within the limited disk or memory space provided by certain application. It likewise reduces the time required for images to be sent over the Internet or downloaded from Web pages [5].

\subsection{Types of image compression}

There are a few different ways in which image files can be compressed. For Internet utilize, the two most basic compressed graphic image formats are the JPEG format and the GIF format. The JPEG method is all the more regularly utilized for photographs, while the GIF method is ordinarily utilized for line art and different images in which geometric shapes are relatively simple. Different methods for image compression incorporate the utilization of fractals and wavelets. There are mainly two types of image compression techniques discussed below [6]:

\subsubsection{Lossy Image Compression:}

In this sort of image compression there is a loss of information. In the event that the compressed image is decompressed then it won't be identical to original image yet close to it. Various lossy compression techniques are Transformation coding, Vector quantization, Fractal coding.

\subsubsection{Lossless Image Compression:}

In this kind of image compression there is no loss of information. In the event that the compressed image is decompressed then it will be identical to the original image. There are various lossless image compression techniques are Run length encoding, Huffman encoding, Area coding, Data folding [7].

\section{LITERATURE REVIEW}

Mansour Nejati, et.al, (2016) proposed in this paper [8] a boosted dictionary learning structure to develop an ensemble of complementary particular dictionaries for sparse image representation. Based on the proposed ensemble model, we then build up another image compression calculation utilizing boosted multiscale dictionaries learned as a part of the wavelet domain. Our calculation is assessed for compression of natural images. Trial comes about exhibit that the proposed calculation has better rate distortion execution as contrasted and a few contending compression strategies including explanatory and learned dictionary schemes.Christian Rathgeb, et.al, (2016) proposed in this paper [9] the influence of different cutting edge image compression principles on identification and ear recognition algorithms. Evaluations directed on an uncompressed ear database are considered regarding different stages in the handling chain of an ear recognition framework where compression might be connected, speaking to the most applicable forensic situations. 
This work explored the feasibility and limitations of ear recognition affected by image compression. Based on test comes about got for three different discovery algorithms and four feature extraction techniques it is presumed that, ear recognition may be feasible within the sight of serious image compression relying upon a few factors Azam Karami, et.al, (2016) proposed in this paper [10], another lossy compression strategy for hyperspectral images that intends to optimally compress in both spatial and spectral domains and at the same time minimizes the impact of the compression on linear spectral unmixing execution.The proposed calculation achieves a superior execution (higher SNR variance and littler MSE) in comparison with two cutting edge compression algorithms, especially at high CRs. A quick rough strategy that fixes the center tensor measurements is presented also. In future work, the point is to decrease the computational complexity of the proposed calculation.

Jianquan Yang, et.al (2015) proposed in this paper [11], a hypothetical examination on the variety of local variance brought about by JPEG compression is introduced. To start with, the desire of intensity variance of $8 \times 8$ non-covering blocks in a JPEG image is inferred. Second, some fascinating properties that portray the conduct of the local variance under different degrees of JPEG compression are talked about. Last, both simulation and tests are performed to check our derivation and examination. The hypothetical examination exhibited in this paper gives some new bits of knowledge into the conduct of local variance under JPEG compression. Besides, it can possibly be utilized as a part of a few ranges of image handling and examination, for example, image upgrade, image quality evaluation and image filtering .

R.O. Preda et.al, (2015) proposed in this paper [12], a watermarking-based image verification plan in the discrete cosine change (DCT) domain hearty to JPEG compression. The twofold validation code is created from a pseudorandom grouping based on a mystery key and a block-dependent feature, securing the plan against cut-and-glue attacks. Contrasted and other existing schemes, the proposed calculation achieves better execution with respect to false positive and false negative discovery rates and in segregating malicious attacks from JPEG compression. The plan achieves high image quality of around $45 \mathrm{~dB}$ and low false positive and false negative identification rates contrasted and other existing image verification procedures. Jianquan Yang, et.al, (2014) proposed in this paper [13], a compelling error-based measurable feature extraction plan. Trial comes about on three image databases with different quality factors have shown that the proposed technique can essentially beat the cutting edge strategy. Based on the investigation, 13-dimensional errorbased factual features were removed from rounding and truncation error blocks independently. At last, with the separated features, the SVM classifier is connected for identifying double JPEG compression.

\section{WDR ALGORITHM}

a. Initialization: The first step is assigning a scan order. For instance, for an image that contains $P$ number of pixels, the scan order is a one-to-one and onto mapping $=\mathrm{Xk}$, for $\mathrm{k}=1,2 \ldots \mathrm{P}$ in between the wavelet coefficient and a linear ordering (Xk). Across the sub bands that are from higher to lower levels, the scan order is in the zigzag manner. An initial threshold T0 is selected such that all the transform values satisfy that $|\mathrm{Xk}|<\mathrm{T} 0$ after the scanning order is made. Also, it is to be ensured that at minimum, one transform value must satisfy the condition $|\mathrm{Xk}|>=\mathrm{T} 0 / 2$

b. Significance pass: It is checked within this step whether the transform values are greater than or equal to the threshold values or not. Further, with the help of various reduction methods of Tian and Wells, the index values are encoded. A binary coding of the numerous steps that are to be started from index of the last significant value till the index of the current significant value are included within this difference reduction method.

c. Refinement pass: The refined bits are generated with the help of standard bit-place quantization process within this step. Every single refined value provides enhanced approximation of the exact transform value within this process

\section{PROPOSED METHODOLOGY}

The proposed technique increase the compression ratio using the greedy algorithm. The proposed technique consist of steps described below:-

1. Discrete Coefficient Transformation (DCT): The DCT technique is the coefficient based transformation in which the colored features of the input image are been analyzed and processed. In the proposed technique the image indexing is being done according to the pixel value of input image. To apply vector quantization the coefficients of the input image are calculated which the color intensity values are and in the last step the matrix of color intensity values is generated by taking mean of the pixels.

2. Vector Quantization: The vector quantization of the input value is generated from the mean matrix which is generated in the previous step. In the vector quantization, three steps are followed. In the first step, the gray scale pixels of the input image are divided to analyze individual gray scale pixels. In the second step, the RGB pixels are divided to analyze individual part of the pixel. In the last step, the DB values of the pixels are generated to generate final compressed image.

3. DCT and Image Masking: In the last step, the technique of DCT and image masking is being applied in which the pixels which have least importance are removed from the image. The importance of the pixels of the image is being analyzed through the DB values generated in the vector quantization. This leads to generation of final compressed image which has less size than the input image. 


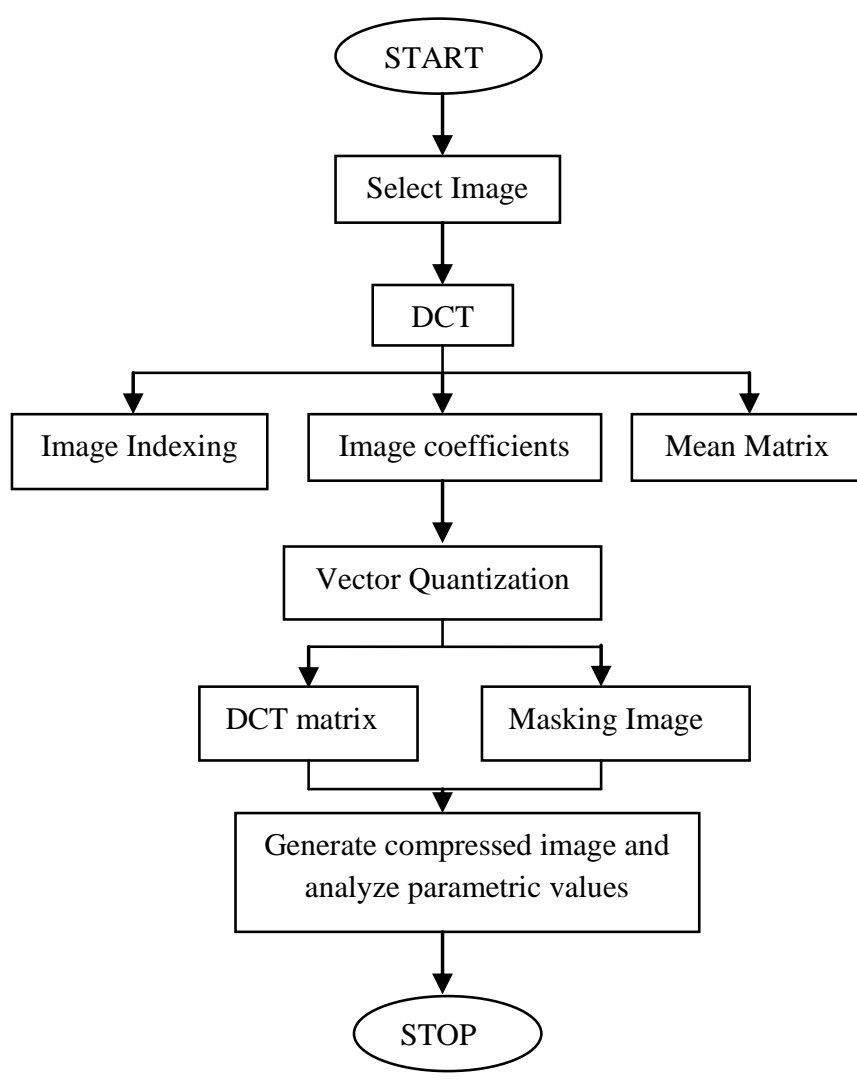

Fig 1: Proposed Flowchart

\section{RESULT \& DISCUSSIONS}

The proposed technique has been implemented in MATLAB by taking the dataset of 10 image which are in the grayscale.

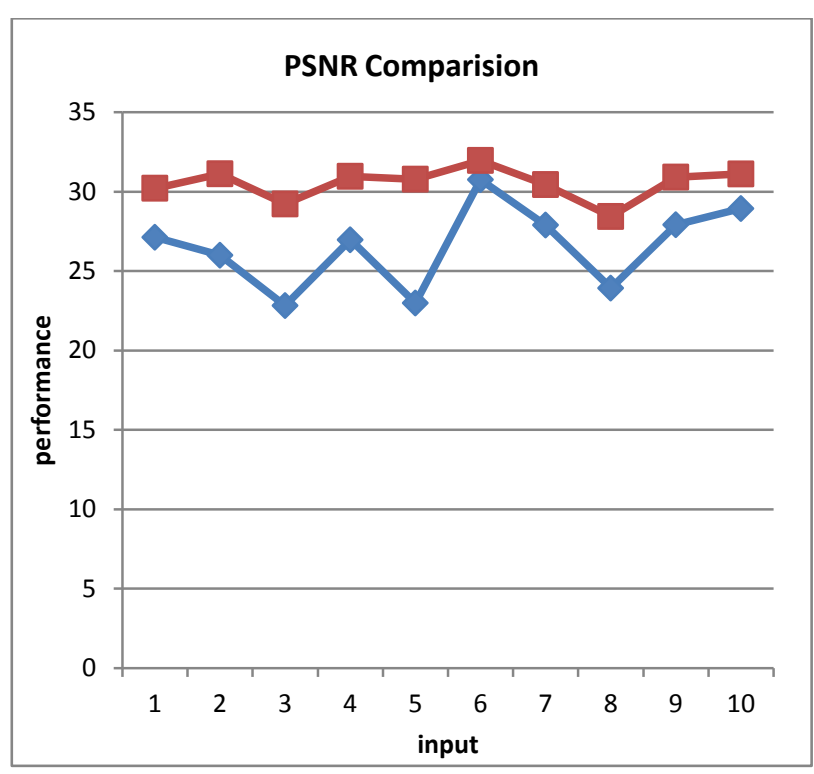

Fig 2: PSNR Comparison

As we know that PSNR, is an engineering term for the ratio between the maximum possible power of a signal and the power of corrupting noise that affects the fidelity of its representation so its value should be high.

Here the red line represent the proposed technique and blue line represents the existing technique As shown in figure 2, the PSNR value of the proposed and existing technique is been compared and it is been analyzed that PSNR is increased at steady rate due to greedy behavior of the algorithm. .It can be seen that PSNR using the improved technique is increased. As compared to the existing algorithm so the algorithm has becomes better.

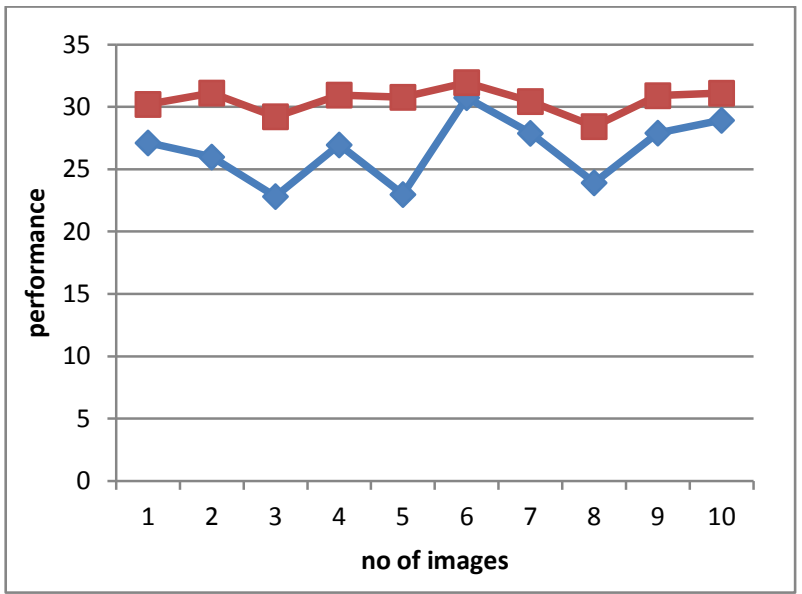

Fig 3: Compression ratio comparison

As Compression ratio is defined as the ratio between the uncompressed size and compressed size so its value should be higher.

Here the red line represents the compression ratio of proposed algorithm and blue line shows the compression ratio of exiting algorithm and in the figure 3 , it can be seen that the compression ratio of the proposed technique is more as compared to exiting algorithm..

Thus this improved algorithm is better since its compression ratio is higher.

\section{CONCLUSION}

The image compression is the technique in which size of the image is reduced by removing the unwanted pixels from the image. The WDR is the efficient technique in which the whole image is divided into small matrix and matrix which has dissimilar properties are removed from the image.

In this work, the greedy algorithm is been proposed in which tree like structure is used where leaf metrics are removed from the images. The result of the proposed algorithm is compared with existing WDR algorithm and it is been analyzed that PSNR is increased and the ,compression ratio is also increased. Thus the improved algorithm is better in terms of performance.

\section{REFERENCES}

[1] Sung Kyu Lee, Sung Gyun Kim, and Young Hwan Kim," Multimode Image Compression Algorithm Employing Multiple-Choice Knapsack Problem-based Encoding Mode Selection", 2015, IEEE

[2] Mansour Nejati, Shadrokh Samavi, Nader Karimi, S. M. Reza Soroushmehr, and Kayvan Najarian," Boosted Dictionary Learning for Image Compression", 2016, IEEE

[3] Christofer Schwartz, and Marcelo da Silva Pinho," Remote-Sensing Image Compression Using Embedded Multicore Platforms With Energy Consumption Measurement", IEEE GEOSCIENCE AND REMOTE SENSING LETTERS, 2015, IEEE 
[4] Cuiping Shi, Junping Zhang, and Ye Zhang," A Novel Vision-Based Adaptive Scanning for the Compression of Remote Sensing Images", IEEE TRANSACTIONS ON GEOSCIENCE AND REMOTE SENSING, 2015

[5] R. Flamary, M. Fauvel, M. Dalla Mura, and S. Valero," Analysis of Multitemporal Classification Techniques for Forecasting Image Time Series", 2015, IEEE GEOSCIENCE AND REMOTE SENSING LETTERS, Vol. 12, No. 5

[6] Amin Zehtabian, and Hassan Ghassemian," An Adaptive Pixon Extraction Technique for Multispectral/Hyperspectral Image Classification", 2015, IEEEGEOSCIENCE AND REMOTE SENSING LETTERS, Vol. 12, No. 4

[7] Gang Li, Xiao Li, Sheng Li, Huang Bai, Qianru Jiang, and Xiongxiong He," Designing Robust Sensing Matrix for Image Compression", IEEE TRANSACTIONS ON IMAGE PROCESSING, VOL. 24, NO. 12, DECEMBER 2015
[8] Mansour Nejati, Shadrokh Samavi, Nader Karimi, S. M. Reza Soroushmehr, and Kayvan Najarian," Boosted Dictionary Learning for Image Compression", 2016, IEEE, 1057-7149

[9] Christian Rathgeb, Anika Pflug, Johannes Wagner, Christoph Busch," Effects of image compression on ear biometrics", 2016, IET Biom., 2016, Vol. 5, Iss. 3, pp. 252-261

[10] Azam Karami, Rob Heylen, and Paul Scheunders," Hyperspectral Image Compression Optimized for Spectral Un mixing", 2016, IEEE TRANSACTIONS ON GEOSCIENCE AND REMOTE SENSING, 0196-2892

[11] Jianquan Yang, Guopu Zhu, and Yun-Qing Shi," Analyzing the Effect of JPEG Compression on Local Variance of Image Intensity", 2015, IEEE, 1057-7149

[12] R.O. Preda and D.N. Vizireanu," Watermarking-based image authentication robust to JPEG compression", 2015, ELECTRONICS LETTERS, Vol. 51 No. 23 pp. 1873-1875 